Ethiopian Journal of Environmental Studies \& Management 8(3): 330 - 341, 2015.

ISSN:1998-0507

doi: http://dx.doi.org/10.4314/ejesm.v8i3.10

Submitted: January 25, 2015

Accepted: April 02, 2015

\title{
CANONICAL CORRELATION ANALYSIS OF HEALTH STATUS AND SOCIO-ECONOMIC CHARACTERISTICS OF RURAL HOUSEHOLDS IN AKWA IBOM STATE, NIGERIA
}

\author{
*UDOH, U.P. ${ }^{1}$ AND ESSIEN, A.U. ${ }^{2}$ \\ ${ }^{1}$ Department of Architecture, University of Uyo, Uyo, Nigeria \\ ${ }^{2}$ Department of Geography and Natural Resources Management, University of Uyo, Uyo, \\ Nigeria
}

\begin{abstract}
The study examines the patterns of linkage between households' health status and their socio-economic characteristics in rural Akwa Ibom State using canonical correlation analysis. Data measuring nine socio-economic variables and six health measures were collected from 540 randomly sampled households in 90 villages using the Socio-Economic and Health Questionnaire (SHQ) as the main instrument of data collection. Though the households' health and socio-economic profiles were found to be abysmally low, the result of the canonical correlation revealed three fundamental ways the peoples' health status are linked to socio-economic characteristics. Three canonical functions representing three models were generated. The first canonical function $(r 1=0.99 ; p \leq 0.05)$ demonstrate the link between respiratory health and housing condition; the second canonical function ( $r 2=0.96 ; p \leq 0.05)$ illustrates an inverse relationship between water-related diseases and household sanitation standard while the third canonical function $(r 3=0.93 ; p \leq 0.05)$ indicated a strong reciprocal relationship between the peoples' healthcare behavior and income/occupational structure. On the whole, it was concluded that rural households share a common variance on health and socio-economic status. The study therefore proposed a new paradigm for achieving accelerated and sustainable health standards for the rural population in developing countries that is hinged on total rural socio-economic transformation.
\end{abstract}

Key Words: Rural, Health, Household, Correlation, Analysis, Socio-economic

\section{Introduction}

Improving health conditions and the general socio-economic standard of rural people has been the major thrust of most developmental efforts of governments, nongovernmental organizations and development partners at the local and international levels. The United Nations had clearly articulated in her Millennium Development Goals (MDGs) the objective of halving the world's poor and reducing infant mortality by the year 2015 . This has attracted scholarly research on the relationship between Socio-Economic
Status (SES) and health conditions of population in both developed and developing countries (Michael, 2004).

In developed countries like the United States, studies have generated enormous empirical evidence linking the people's socio-economic status with health conditions. Adler and Newman (2002) found that health determinants namely: healthcare, environmental exposure, and health behavior, shared an underlying factor with socio-economic status, thereby justifying the application of the socioeconomic status paradigm for reducing

*Corresponding Author: Udoh, U.P.

Email: usenudoh4favour@gmail.com 
health disparities among Americans. Furthermore, studies by McGinnis and Foege (1995) revealed that households with lower socio-economic status were significantly associated with higher mortality. A wide range of health problems including low birth weight, respiratory disease, hypertension, arthritis, diabetes and cancer were also linked to socioeconomic components such as income, education or occupation (Mare, 2000).

In less developed countries, studies have also documented the relationship between socio-economic differentials and health status of the population. In a crosssectional Demographic and Health Survey (DHS) from twenty developing countries, Bicego and Ahmed (1996) found that mortality risk of under fives born to uneducated women were twice as high as those born to women with a secondary education. Gwatkin et al. (2000) described mortality, nutrition and morbidity differences in Tanzania based on socioeconomic characteristics of households. Studies in Zimbabwe also revealed that stunting underweight and occurrence of diarrhea, cholera and respiratory symptoms varied according to the socio-economic status. It was noted that being in the lowest socio-economic status increased the risk of being underweight for children by about three times compared to those in the highest socio-economic group (Woelk and Chikuse, 2000).

In a study of 22 malarial countries in Africa, Filmer (2000) found little difference in rates and risk of fever among the poor and least poor, and that the poorest had a much smaller likelihood of obtaining suitable treatment. In a similar study in rural Tanzania, low poor/ least poor inequities across a broader array of childhood illness and health interventions were observed based on a principal component analysis of household data from a cross-sectional survey (Schellenberg et al., 2003).

In Nigeria and Akwa Ibom State in particular, there is dearth of information on the relationship between health status and socio-economic characteristics especially among rural dwellers. The purpose of this study therefore is to explore the pattern of relationship between household's health status and their socio-economic characteristics in rural Akwa Ibom State using canonical correlation analysis.

\section{Study Area}

Akwa Ibom State is located in the southern coast of Nigeria. The State is bounded by Rivers, Abia and Cross River State and the Republic of Cameroun to the southwest, north, east and southeast respectively, while the Bight of Bonny borders the State to the south (Figure 1). The State has 31 Local Government Areas. According to the 2006 National Population Census records, Akwa Ibom had a total population of $3,920,208$ persons out of which 87.89 percent constituted rural population while 12.11 percent formed the urban population (NPC, 2006). 


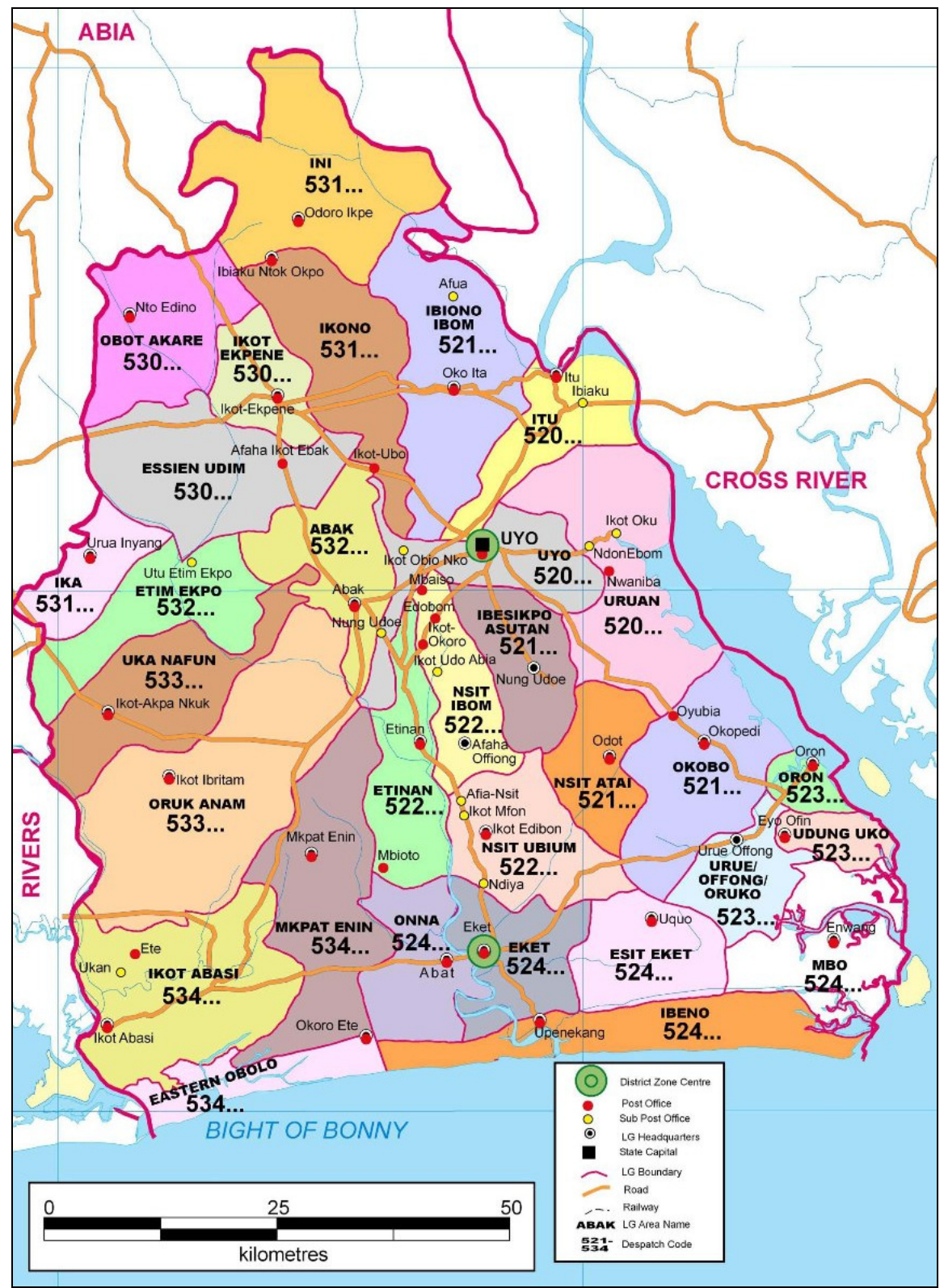

Figure 1: $\quad$ Map of Akwa Ibom State

\section{Methodology}

\section{Research Design and Data Collection}

The data used in this study were collected as an integral part of Demographic and Health Survey (DHS). A cross-sectional survey based on a probability sample of 540 households in 90 rural communities in Akwa Ibom State was carried out in 2012. The selection of sample communities was facilitated by obtaining a base map of Akwa Ibom State on a scale of $1 \mathrm{~cm}$ to $2.5 \mathrm{~km}$, and dividing same into quadrants of $1.6 \mathrm{~km}^{2}$ which was clearly and serially numbered. A total of 480 quadrants were produced. However, 90 quadrants representing $18.75 \%$ was selected using a table of random numbers. The grid map contained the names of communities in each quadrant. In Nigeria, rural communities are defined numerically by a population below 20,000 people (NPC, 2006). With this in mind, communities with population of 20,000 and above were excluded. Furthermore, where a quadrant contains two or more rural communities, 
only one was randomly selected from such quadrant.

Finally, six households from each community were systematically selected to yield a total of 540 households. An average rural community in Akwa Ibom State has sixty households (NPC, 2006), therefore, six households representing ten percent is valid for generalization (Enoh, 2006). The study used a structured questionnaire designated as "the Socio-Economic and Health Questionnaire", (SHQ) that was administered during a face to face interview with heads of households or a proxy respondent from the household. The questionnaire comprised of sections on household socio-economic characteristics and health variables as indicated in Table 1.

Table 1: $\quad$ Household Socio-Economic and Health Measures

\begin{tabular}{lll}
\hline SOCIO-ECONOMIC MEASURES & UNIT OF MEASUREMENT \\
\hline 1 & Income level & High (3), Medium (2), Low (1) \\
2 & Occupation & Public service (3), Business (2), Farming(1) \\
3 & Educational Attainment & Tertiary (3), Secondary (2), Primary (1), No \\
& & formal education (0) \\
4 & House type & Block (3), Mud (2), Thatch (1) \\
5 & Source of water & Borehole (3), Well (2), Stream/River (1) \\
6 & Toilet type & Water Closet (3), Pit Latrine (2), Open Square (1) \\
7 & House condition (roof, wall \& floor) & Good (3), Fairly Good (2), Fair(1), Bad(0) \\
8 & Type of waste disposal & Receptacle (3), Dump Site (2), Bush(1) \\
9 & Crowding & <2persons/Room (3), 2persons/Room (2), $>2$ \\
& & Persons/Room(1) \\
HEALTH MEASURES & UNIT OF MEASUREMENT \\
\hline 1 & Occurrence of Diarrhea & Occurrence (1), Non-Occurrence (0) \\
2 & Incidence of Pneumonia & Occurrence (1), Non-Occurrence (0) \\
3 & Cholera attack & Occurrence (1), Non-Occurrence (0) \\
4 & Access to healthcare & Travel Time To Nearest Health Centre \\
5 & Mode of disease treatment & Orthodox (3), Traditional (2), Spiritual (1) \\
6 & Frequency of treatment & Always (3), Occasionally (2), Rarely (1) \\
\hline
\end{tabular}

These variables have been widely used in socio-economic and health studies. Though some studies limit socio-economic variables to income, occupation and educational attainment (Roberts, 1995; Cadwell, 1980; Mare, 2000), others have applied an extended concept of socioeconomic status to accommodate housing and environmental variables (Omole, 2010; Amstrong et al., 2003; Woelk and Chikuse, 2000). In this study therefore, the researchers carefully selected ten independent variables which are relevant in capturing the socio-economic status of a typical Nigerian rural household.

The inclusion of housing and environmental measures is justified on the grounds that theoretically, housing and environmental factors are surrogate indicators of population socio-economic status in developing countries (Ukpong, 2009; Sahn and Stifel, 2000). Six health variables represented the outcome measure (dependent variable) of the study. The variables captured both issues of mortality, morbidity and healthcare behavior of the rural populace.

\section{Data Analysis}

Canonical correlation analysis (Cancorr) was employed to explore the different ways households in rural areas of Akwa Ibom State share a common variance on socio-economic and health characteristics. Cancorr is a multivariate statistical model that investigates a maximum relationship between two sets of multiple dependent variable and multiple independent variables. In this case, the 
indicators of health status are considered the "multiple dependent variables" whereas the indicators of socio-economic status are considered the "multiple independent variables". Cancorr is the most suitable technique to use when dealing with multiple dependent variables and independent variables, either metric or nonmetric variables (Hair et al., 1998).

The first step of Cancorr is to derive one or more canonical functions. Each function consists of a pair of variates, one as independent variables and the other as dependent variables. The first function extracted accounts for the maximum amount of variance in the two sets of variables, that is, the first pair of canonical variates exhibits the highest intercorrelation possible between the two sets of variables. The second pair of canonical variates derived accounts for the maximum amount of "leftover" variance.

Two criteria were used for selecting the canonical function for interpretation:

Level of statistical significance of the function; and

Magnitude of the canonical correlation.

\section{Results and Discussion Socio-economic and Health Profile of the People}

The socio-economic measures adopted for this study indicated an abysmally low socio-economic status for the study area. As data in Table 2 shows, majority of the respondents (80\%) belong to the low income group who are earning less than eighteen thousand Naira per month. Only $8 \%$ of sample households had an earned income of above eighteen thousand Naira, which is the statutory minimum wage for the Nigerian worker. Occupationally, family household had predominance (69\%) over business (19\%) and public service $(8 \%)$. The highest educational attainments of household heads were: Tertiary (14\%), Secondary $(25 \%)$ and Primary (39\%).
Furthermore, 22\% of household heads had no formal education.

The housing component of the household socio-economic status (SES) captured the variables namely; house type, source of water, toilet type, house condition (in terms of roof, wall and floor condition), type of waste disposal and room crowding. Expectedly, households representing 58\% lived in mud-walled houses, $19 \%$ lived in thatched houses while $20 \%$ are housed in block walled buildings. Additionally, 77\% of households sourced their water from distant streams/rivers $4 \%$ obtained water from dogged well, while $19 \%$ had water supply from boreholes. For toilet type, $63.5 \%$ of households patronize pit latrine; $23.0 \%$ defecates in the open square/ nearby bush while only $13.5 \%$ use the water closet. In terms of house condition (specifically the state of the walls, roof and floor) $25.0 \%$ of households live in fairly good house condition: while $38.4 \%$ inhabits houses with poor roof, wall and floor condition. Data in table 2 also indicates that $77.0 \%$ of households disposed waste in the bush; $19.2 \%$ use the designated dump site while only $3.8 \%$ of the household owned waste receptacles. For crowding, $71 \%$ of households have more than two persons occupy a single room while $10 \%$ have less than two persons per room. Evidence from the above data on rural socio-economic characteristics in Akwa Ibom State confirms previous assertions by Udofia et al. (2012) and Esin (2010) that rural conditions in most part of Nigeria are matters of emergency. With large proportion of the people living below the national minimum wage (of 18000 per month); issues of non-availability of portable water; poor sanitary and housing conditions; rural Nigeria requires a holistic intervention to change their current socioeconomic profile.

Table 2 also contains data indicating the health status of the households. Accordingly, 52.0\% of households reported 
frequent occurrence of pneumonia; $25 \%$ reported Diarrhea; while 23.0\% reported for cholera. Furthermore, the mode of treatment and the frequency of treatment of reported illnesses above varied with households. Whereas 48\% utilize the traditional mode of treatment, only $23 \%$ utilizes the orthodox while $29 \%$ prefer the spiritual mode of treatment. On the whole, only $10 \%$ of households always go for treatment. Majority of households occasionally treat illnesses. In terms of access to healthcare, $67.0 \%$ households spend more than 60 minutes to access healthcare.

Table 2: Household health and socio-economic characteristics in Rural Akwa Ibom State, Nigeria $(n=540)$

\begin{tabular}{|c|c|c|}
\hline Parameter & $\mathbf{N}$ & $\%$ \\
\hline Sample & 520 & 100 \\
\hline \multicolumn{3}{|l|}{ Income level } \\
\hline $\operatorname{Low}(<\# 18,000)$ & 417 & 80 \\
\hline Medium-Low( $\$ 18,000)$ & 60 & 12 \\
\hline Medium-High(> 18,000) & 43 & 8 \\
\hline \multicolumn{3}{|l|}{ Occupation } \\
\hline Public service & 40 & 8 \\
\hline Business & 100 & 19 \\
\hline Farming & 360 & 69 \\
\hline Others & 20 & 4 \\
\hline \multicolumn{3}{|l|}{ Educational Attainment } \\
\hline Tertiary & 70 & 14 \\
\hline Secondary & 130 & 25 \\
\hline Primary & 205 & 39 \\
\hline Non-formal education & 115 & 22 \\
\hline \multicolumn{3}{|l|}{ House type } \\
\hline Block & 120 & 23 \\
\hline Mud & 300 & 58.0 \\
\hline Thatch & 100 & 19 \\
\hline \multicolumn{3}{|l|}{ Source of Water } \\
\hline Borehole & 100 & 19 \\
\hline Well & 20 & 4 \\
\hline Stream & 400 & 77 \\
\hline \multicolumn{3}{|l|}{ Toilet Type } \\
\hline Water closet & 70 & 13.5 \\
\hline Pit latrine & 330 & 63.5 \\
\hline Open square/ bush & 120 & 23.0 \\
\hline \multicolumn{3}{|c|}{ House Condition (roof, wall and floor condition) } \\
\hline Good & 90 & 17.4 \\
\hline Fairly good & 130 & 25.0 \\
\hline Fair & 100 & 19.2 \\
\hline Poor & 200 & 38.4 \\
\hline \multicolumn{3}{|l|}{ Type of waste disposal } \\
\hline Receptacle & 20 & 3.8 \\
\hline Dumpsite & 100 & 19.2 \\
\hline Bush & 400 & 17.0 \\
\hline \multicolumn{3}{|l|}{ Crowding } \\
\hline$<2$ persons/ room & 70 & 10 \\
\hline 2 persons/ room & 120 & 19 \\
\hline
\end{tabular}




\begin{tabular}{lll}
\hline$>2$ persons/ room & 330 & 71 \\
& & \\
Household reporting Diarrhea & 130 & 25.0 \\
Household reporting Pneumonia & 270 & 52.0 \\
Household reporting Cholera & 120 & 23.0 \\
Access to healthcare (travel time to nearest healthcare) & & \\
$<60$ minutes & 50 & 10 \\
60 minutes & 120 & 23.0 \\
$>60$ minutes & 350 & 67.0 \\
Mode of disease treatment & & 23.0 \\
Orthodox & 120 & 48.0 \\
Traditional & 250 & 29.0 \\
Spiritual & 150 & 10.0 \\
Frequency of treatment & & 52.0 \\
Always & 50 & 38.0 \\
Occasionally & 270 & \\
Rarely & 200 & \\
\hline
\end{tabular}

Pattern of Relationship between Linear Combination of the Household Socioeconomic Variables and Linear Combinations of Health Status

Conceptually, the best approach to explore the pattern of relationship between two linearly combined multi-variables such as households' socio-economic factors and health status is by canonical correlation analysis. The essence of the canonical correlation is to derive a linear combination for each of the multivariate data set in such a way that the correlation between the two linear combinations is maximized (Udofia, 2011). Table 3 contains the results of the canonical correlation analysis carried out on all the fifteen variables measuring both households socio-economic and health status.

The steps involved in analysis and interpretation of canonical correlation output on the computer aided software is reasonably straight forward. The first step is to select one or more canonical function that would account for the maximum amount of correlation between the two data sets. Canonical function consists of pairs of variate, one as dependent (criteria) variable and the other as independent (predictor) variable. It is possible for the software to generate canonical functions as much as the number of variables in the smaller data set. However, it is the first canonical function that is of utmost importance to the analysis as it possesses the highest inter-correlation possible between the two data sets. The second, third, fourth and so on are diminished in importance as they take care of the residual variance. For this study therefore as data in table 3 would indicate three canonical functions were selected and used to show three ways households health status are related to socio-economic variables. The choice of three canonical functions out of the maximum number of six extracted was based on the magnitude of the canonical correlation and level of statistical significance of the functions. Accordingly, the $1^{\text {st }}, 2^{\text {nd }}$ and $3^{\text {rd }}$ canonical functions had a canonical correlation of 0.99 ; 0.96 ; and 0.93 respectively (canonical correlation measures the correlation between pairs of canonical variates in the two data sets) and are significant on the Wilk's Lamda, Pillars Trace, LawleyHotelling and Roy's greatest root texts ( $p \geq 0.05$ on all the four texts). 
Table 3: Canonical Structure Loadings for Household Health Status- Socio-Economic Status Interrelationships (Reporting loadings greater than -0.49 and +0.49 on three significant canonical variates)

\begin{tabular}{|c|c|c|c|c|c|}
\hline \multirow[t]{2}{*}{ Variable No. } & \multirow[t]{2}{*}{ Variables } & & \multicolumn{3}{|c|}{ Canonical Function } \\
\hline & & & 1 & 2 & 3 \\
\hline & \multicolumn{5}{|c|}{ Criteria Variable (p): Health Measures } \\
\hline Y1 & \multicolumn{2}{|c|}{ Diarrhea } & 0.28 & $0.61 * *$ & -0.41 \\
\hline Y2 & \multicolumn{2}{|c|}{ Pneumonia incidence } & $-0.59 * *$ & -0.49 & 0.36 \\
\hline Y3 & \multicolumn{2}{|c|}{ Cholera incidence } & -0.23 & $0.77 * *$ & 0.23 \\
\hline Y4 & \multicolumn{2}{|c|}{ Access to healthcare } & -0.22 & 0.43 & $-0.63 * *$ \\
\hline Y5 & \multicolumn{2}{|c|}{ Mode of treatment of disease } & $0.55^{* *}$ & -0.45 & $-0.89 * *$ \\
\hline Y6 & \multicolumn{2}{|c|}{ Frequency of treatment of disease } & 0.14 & -0.28 & $-0.59 * *$ \\
\hline & \multicolumn{2}{|c|}{$\begin{array}{l}\text { Predictor Variables (q): Socio-economic } \\
\text { Status }\end{array}$} & & & \\
\hline $\mathrm{X} 1$ & \multicolumn{2}{|c|}{ Household income level } & -0.18 & 0.49 & $-0.94 * *$ \\
\hline $\mathrm{X} 2$ & \multicolumn{2}{|c|}{ Occupation of head of household } & 0.06 & 0.18 & $-0.69 * *$ \\
\hline $\mathrm{X} 3$ & \multicolumn{2}{|c|}{ Educational level of head of household } & $0.87 * *$ & 0.32 & 0.41 \\
\hline $\mathrm{X} 4$ & \multicolumn{2}{|c|}{ House type } & -0.26 & 0.40 & -0.32 \\
\hline X5 & \multicolumn{2}{|c|}{ Source of water } & -0.49 & $-0.84 * *$ & 0.39 \\
\hline X6 & \multicolumn{2}{|c|}{ Toilet type } & 0.39 & $-0.71 * *$ & 0.29 \\
\hline $\mathrm{X} 7$ & \multicolumn{2}{|c|}{ House condition } & $0.60 * *$ & 0.47 & -0.44 \\
\hline $\mathrm{X} 8$ & \multicolumn{2}{|c|}{ Waste disposal method } & -0.44 & $-0.56 * *$ & -0.16 \\
\hline X9 & \multicolumn{2}{|c|}{ Room crowding } & $0.78 * *$ & 0.50 & -0.21 \\
\hline & \multicolumn{2}{|c|}{ Canonical correlations } & 0.99 & 0.96 & 0.93 \\
\hline \multicolumn{6}{|c|}{ Test of Significance of all Canonical Correlations } \\
\hline Test text & Statistics & df1 & df 2 & $\mathrm{~F}$ & s.g. \\
\hline $\begin{array}{l}\text { Wilk's } \\
\text { Lamda }\end{array}$ & .0000103 & 54 & 19 & 3.123 & $.003^{*}$ \\
\hline $\begin{array}{l}\text { Pillai's } \\
\text { trace }\end{array}$ & 4.05902 & 54 & 48 & 1.855 & $.001 *$ \\
\hline $\begin{array}{l}\text { Lawley- } \\
\text { hotelling } \\
\text { trace }\end{array}$ & 128.12 & 54 & 8 & 3.163 & $.004 *$ \\
\hline $\begin{array}{l}\text { Roys } \\
\text { Greatest } \\
\text { root }\end{array}$ & 103.19 & 9 & 8 & 91.724 & $.000 *$ \\
\hline
\end{tabular}

The three canonical functions represent models that explain the pattern of linkage between households' socio-economic variables and health status. Based on the canonical structure loadings displayed in table 3 , the characteristics of the three canonical functions (models) are presented below:

\section{Characteristics of Canonical Function I}

The first canonical function reveals the highest significant correlation between households health status and their socioeconomic characteristics $(\mathrm{r} 1=0.99, \mathrm{p} \leq 0.05)$. however using a cut-off point of 0.50 the link between the health variables and socioeconomic variables was assessed on the criteria data set, Pneumonia occurrence (y2) achieved a high loading (-0.59) alongside mode of disease treatment (y6) with a canonical loading of 0.55 . On the predictor data set, the educational level of head of household (X3) loaded highly 
(0.87) together with house condition (X4) 0.60 and room crowding (X9) having loading of 0.78 Specifically, the joint structure reveals the link between pneumonia occurrence, house condition and also room crowding. The inverse relationship means that pneumonia occurrence is sensitive to housing conditions and is likely to decline with improved housing conditions with reference to reducing room crowding and improving roof, wall, and floor conditions of the house. Furthermore, the positive correlations between educational level of head of household (X3) and mode of disease treatment (Y5) reveals how knowledge acquisition can interfere with health decisions particularly in terms of preferred mode of disease treatment. In rural Nigeria where low levels of educational attainment exist alongside high levels of illiteracy, the dominant mode of disease treatment appears to favor the traditional and spiritual modes (Table 2). With further exposure to formal education rural household would be able to appreciate and utilize modern modes of healthcare. Health campaigns and programs including efforts at achieving the health components of the MDGS would continue to yield marginal results except the educational level of the rural people is improved. On the whole, Canonical function 1 demonstrate the link between respiratory health and housing condition as well as the relationship between health decisions and level of one's knowledge of healthcare. The model representing the $1^{\text {st }}$ canonical correlation function is shown graphically in Figure 1 below.

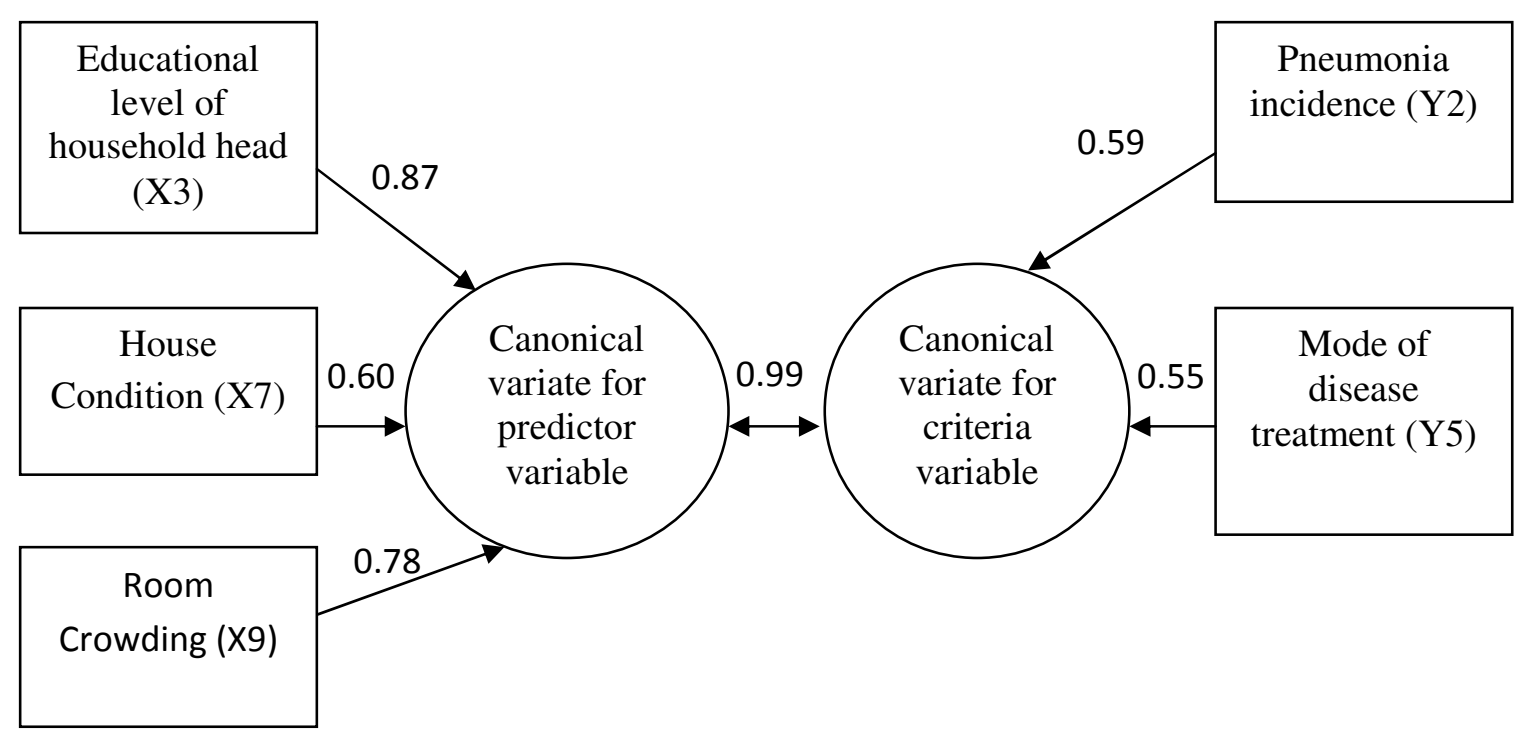

Figure 2: Canonical Function I showing the link between households' health status and socioeconomic characteristics

\section{Characteristics of Canonical Function II}

The second canonical function having a relatively high canonical correlation of 0.96 $(\mathrm{P} \leq 0.05)$ reveals the link between disease incidence and certain households environmental variables. Specifically, this correlation shows the significant inverse relationship between diseases related to water and hygiene e.g. Diarrhea (0.61), cholera (0.77) and those household characteristics associated with hygiene and sanitation e.g. source of water (-0.84), toilet type $(-0.71)$ and waste disposal method (-0.56). The inverse relationship between these set of 
variables shows how sensitive diarrhea and cholera are to unhygienic and unsanitary conditions. In other words, the incidence of diarrhea and cholera appears to increase in households characterized with the use of unsanitary toilet type, unhygienic waste disposal method and impure source of water.

The rural population in Nigeria is bedeviled with the problem of poor access to safe potable water. Majority of rural household depends solely on water sourced from highly polluted streams and rivers and in some cases from untreated borehole water (James and Essien, 2013). Essentially, canonical function II provides the model for improving health status for rural household. This can be done by institutionalizing those polices that would interfere with the environmental components of household characteristics. Particularly, effort towards improving the source of domestic water supply, quality of waste disposal methods and toilet facilities can go along way to undermine the incidence of cholera and Diarrhea. The graphical expression of canonical function II is shown in figure 2 below.

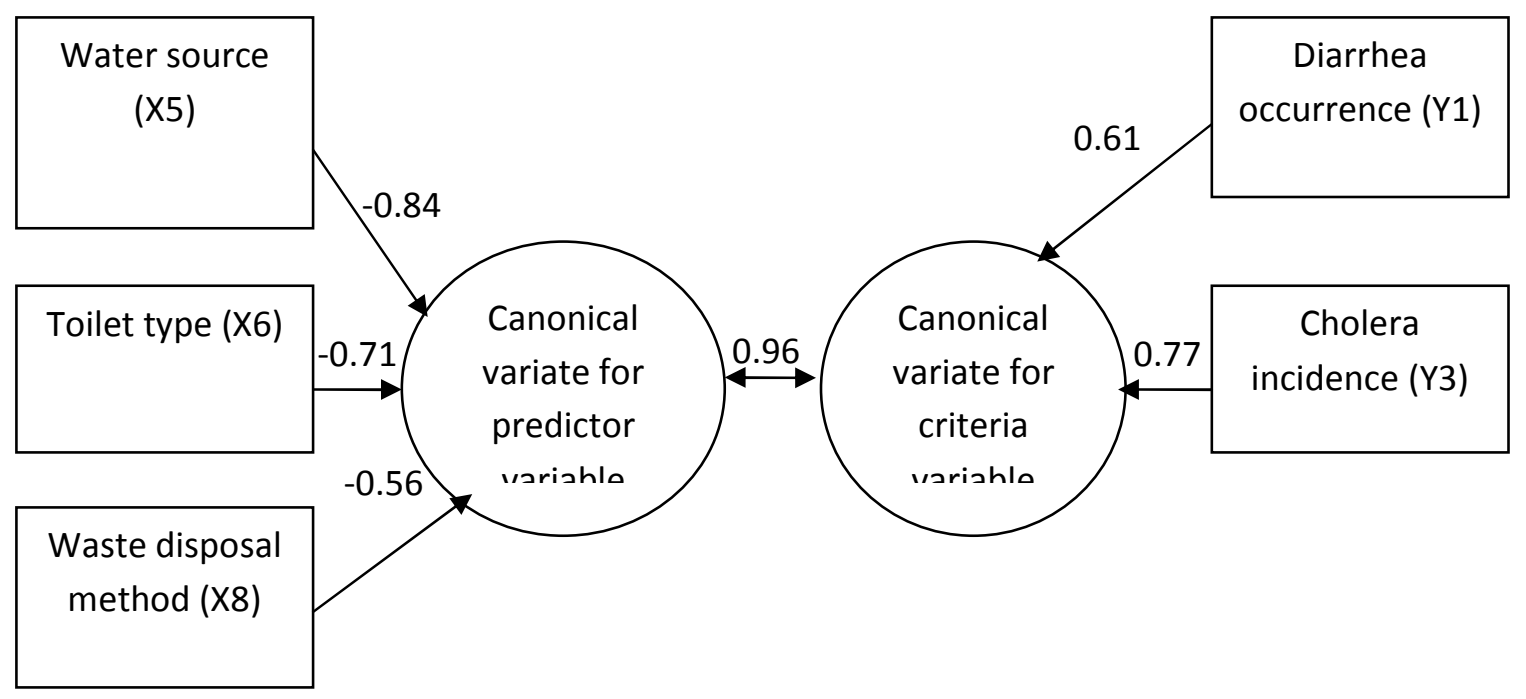

Figure 3: Canonical Function II showing the link between households' health status and socio-economic characteristics

\section{Characteristics of Canonical Function III}

The third canonical function with correlation of 0.93 which is significant at 0.05 significance level indicates a strong reciprocal relationship between the variables associated with household healthcare behavior such as mode of disease treatment (-0.89), frequency of treatment (-0.59) and access to healthcare (-0.63) with the core economic characteristics of household namely: income level (-0.94) and occupation of household head (-0.69). Aside from variables such as the educational attainment of head of household (as already highlighted in canonical function I), income here proves to be a major determinant of healthcare behavior among rural people by achieving a high negative loading on canonical function III. In other words, canonical function III demonstrates the fact that variables underlying households' healthcare behavior share a common variance with the core economic variables. Theoretically therefore, healthcare behavior among rural households can be modified and enhanced through deliberate attempt at improving the financial base alongside the occupational and knowledge base of the rural people. With dwindling agricultural fortune, rural people are bound to suffer financial setbacks except effort at diversifying rural economy and creating non-farming income is intensified. Figure 3 shows the graphical representation of canonical function III. 


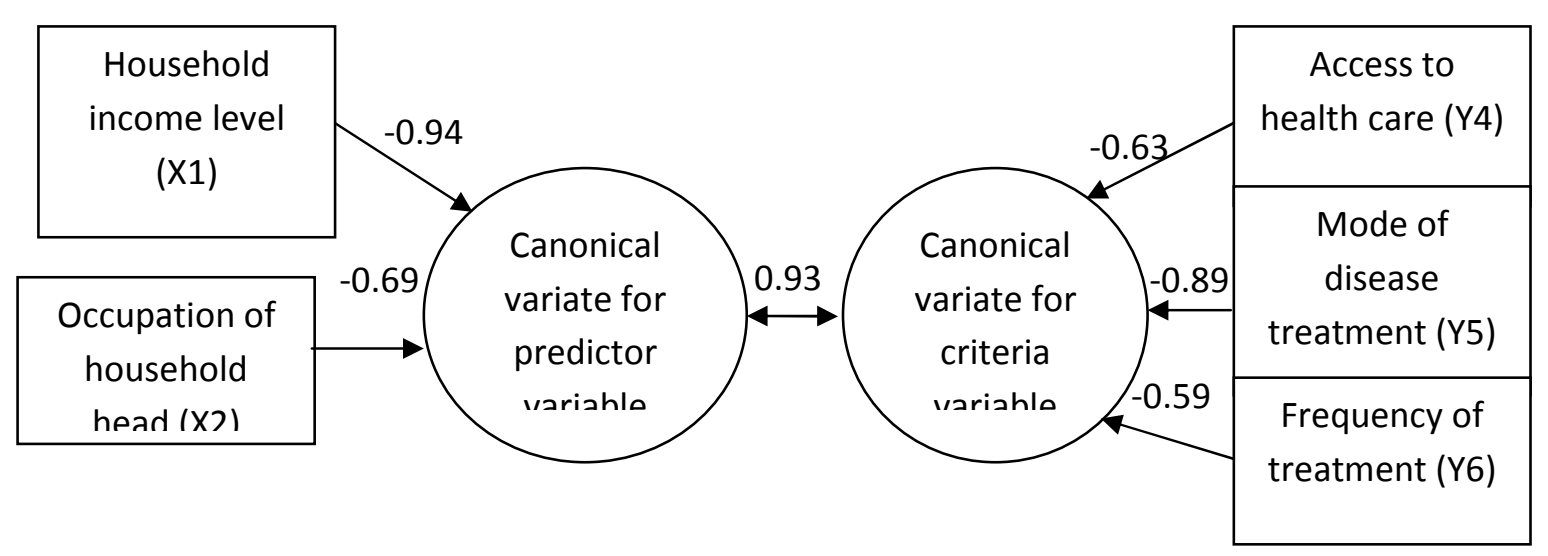

Figure 4: Canonical Function III showing the link between households' health status and socio-economic characteristics

\section{Conclusion}

The research has focused upon the assessment of household's health status and socio-economic characteristics in rural Akwa Ibom state, Nigeria. The various ways through which the health status of the people is related to their socio-economic characteristics has been explored using the canonical correlation analysis. The low socio-economic profile observed among the sampled households revealed the state of under development and low human capital formation evident in rural Nigeria in general. Illiteracy, poverty and limited access to safe drinking water are the major disadvantages of the sampled rural households.

The health status of the people was found to be unacceptably below standard. In as much as the disease burden remain obviously high, access to modern healthcare remained problematic as majority of the people spend significant travel time to access nearby health facility. Furthermore, the healthcare behavior of most of the people remained largely primitive as only negligible proportion of sampled household patronize orthodox healthcare.

The result of canonical correlation analysis revealed three important ways household health status is linked to their socio-economic characteristics. The first pattern showed a common variance between a set of respiratory symptoms and housing conditions; the second captured the relationship between common diseases related to water quality/ hygiene and household environmental variables associated with sanitation while the third demonstrated the link between a set of variables defining household healthcare behavior and household income/occupational structure. These three models highlighted above could serve as theoretical framework that can underpin any genuine effort by stakeholders to improve rural health systems. In general, the findings of this study serve as a "wake up call" for rural developers to focus priority on those policies and programs that seek to transform the socio-economic standard of rural people. Specifically, those policies that would diversify the economic base of the rural enclaves and improve the educational profile of the rural man are highly recommended. As the findings of this study presupposes, such efforts would trigger a multiplier effect then spans through the health status of the people. 


\section{References}

Adler, N. and Newman, K. (2002). "Socio- Economic Disparities in Health: Pathways and Policies". Health Affairs, 21(2): 60- 75

Bicego, G. and Ahmed, O.B. (1996), Infant and Child Mortality Demographic and Health Surveys Comparative Studies No. 20. Calverton, MD: Macro International

Esin, J.O. (2010). "Spatial Analysis of Rural Poverty in Akwa Ibom State". Unpublished Ph.D Thesis, University of Uyo, Uyo, Nigeria

Filmer, D. (2002). Fever and its treatment among the more and more and more less poor in Sub- Saharan Africa. World Bank Development Economics Research Group Working Paper 2789.

Gwatkin, D., Rutstein, S., Johnson, K., Pande, R. P. and Wagstaff, A. (2002). Socio- Economic differences in Health, Nutrition and Population. HNP Poverty Thematic Group of the World Bank

Hair, J., Anderson, R. Tatham, R., and Black, W. (1998). Multivariate Data Analysis (5th Ed.) New Jersey: Prentice-Hall

James, E. and Essien, A. (2012). Emerging Issues in Rural Water Supply: Case Study in Akwa Ibom State, Nigeria. Journal of Environmental Design, 7(2): 114-120

Manly, B. (1994), Multivariate Statistical Methods: A Primer, 2nd Edition. Chapman and Hall, London

Mare, R.D. (2000). Socio-Economic Factors, Health Behaviour and Mortality: Results from a Nationally Representative Prospective of US Adults. Journal of American Medical Association, 279(2): 1703- 1708

McGinnis, J.M. and Foege, W.H. (1995). Actual Cause of Death in the US. JAMA 270(18): 57- 62
NPC (2006). Final Census Report, 2006. Federal Republic of Nigeria

Omole, F. (2010). An Assessment of Housing Condition and Socioeconomic Status of Slum Dwellers in Akure, Nigeria. Contemporary Management Resources 6(4): 273290.

Ogeke, L.A. (2001). Statistics and its Applications in the Sciences. Nigerian Universities Inaugural Lectures Series. National Universities Commission, Abuja, Nigeria

Robert, S. (1995). Socio-Economic Position and Health: The Independent Contribution of Community SocioEconomic Context. Annual Review Sociology, 25: 489- 516

Sahn, D.E. and Stigel, D. (2000). "Assets as a measure of household welfare in developing countries". Unpublished Paper. Cornell University

Schellenberg, J., Victoria, C., Mushi, A., de Savigny, D. and Schellenberg, D. (2003). Inequities among the very poor: Healthcare of Children in Rural Tanzania. Tanzania IMCI MCE Baseline Household Study Group

Udofia, E.P. (2011), Applied statistics with Multivariate Methods. Enugu: Immaculate publishers Ltd.

Udofia, E.P., Atser, J. and Ikurekong, E.E. (2013). Linear Discriminant Analysis of Multiple Groups in Rural Settlements of Akwa Ibom State, Nigeria. Journal of Rural Development, 32 (2): 121-138

Ukpong, I.E. (2009). Perspectives on Environmental Management. Enugu: Immaculate publishers Ltd.

Woelk, G. and Chikuse, P. (2000), "Using Demographic and Health Surveys (DHs) Data to Describe Intra Country Inequalities in Health Status in Zimbabwe" - Paper presented at the EQUINET Conference, Mid-Rand, South Africa 